\title{
Leadership \& Professional Development: Engaging Patients as Stakeholders
}

\author{
Stephanie AC Halvorson, MD, FACP*, Honora Englander, MD
}

Division of Hospital Medicine, Oregon Health \& Science University, Portland, Oregon.

"Nothing about us without us" (Latin: "Nihil de nobis, sine nobis")

Hospitalists are at the forefront of decisions, innovations, and system-improvement projects that impact hospitalized patients. However, many of our decisions-while centered on patient care-fail to include their perspectives or views.

In his book Total Leadership, Stewart Friedman describes the importance of identifying and engaging key stakeholders. ${ }^{1}$ Friedman exhorts leaders to engage stakeholders in conversations to "confirm or correct your current understanding of stakeholder expectations." In other words, instead of assuming what stakeholders want, ask and verify before proceeding.

Although hospitalists frequently include stakeholders such as nurses, pharmacists, and therapists in system-improvement initiatives, engaging patients is less common.

Why do we omit patients as stakeholders? There are considerable barriers to seeking patient input. The busy hospital environment or the acuity of a patient's illness may, for instance, limit engagement between hospital caregivers and patients. Further, the power imbalance between physicians and patients may make it uncomfortable for the patient to offer direct feedback.

However, the importance of patient input is increasingly recognized by researchers. For example, community-based participatory research "involves community members or recipients of interventions in all phases of the research process." 2 Similarly, we believe hospitalists should engage patients when designing new clinical initiatives.

Examples from some institutions provide further support of this concept. The Dana Farber Cancer Institute created a patient and family advisory council in response to the loss of trust over errors and in the face of community outrage over an impending joint venture. While the scope was initially limited to the collection of feedback regarding patient satisfaction and preferences, the council evolved to become an integral part of organizational decision making. Patient contributions were subsequently assimilated into policies, continuous improvement teams, and even search committees. Additional benefits included patient-generated initiatives such as "patient rounds. " 3 Specifically soliciting input from hospitalized patients to inform hospital-based interventions may be uncommon, but this practice holds the potential to yield vital insights. ${ }^{4}$

*Corresponding Author: Stephanie Halvorson, MD FACP; Email: halvorss@ ohsu.edu; Telephone: 503-494-9487.

Received: November 4, 2019; Accepted: November 5, 2019

๑ 2020 Society of Hospital Medicine DOI 10.12788/jhm.3353
We have experienced this benefit at our institution. For example, before implementing an inpatient addiction medicine consult service, we asked hospitalized patients struggling with addiction about their needs. The patient voice highlighted a lack of trust for hospital providers and led directly to the inclusion of peer-recovery mentors as part of the consulting team. ${ }^{5}$

Many organizations, including our own, have instituted a patient/family advisory committee comprising former patients and family members who participate voluntarily in projects and provide input. This resource can serve as an excellent platform for patient involvement. At the University of Michigan, the patient and family advisory council provides input on every major institutional decision, from the construction of a new building to the introduction of a new clinical service. This "hardwired" practice ensures that patients' voices and views are incorporated into major health system decisions.

In order to engage patients as stakeholders, we recommend: (1) Be sensitive to the power imbalance between clinicians and patients and recognize that hospitalized patients may not feel comfortable providing direct feedback. (2) Familiarize yourself with your institution's patient/family advisory committee. If one does not exist, consider soliciting responses from patients via interviews and/or postdischarge surveys. (3) Deliberately seek the opinions, experience, and values of patients or their representatives. (4) For projects aimed at improving patient experience, include patients among your key stakeholders.

Involving patients as stakeholders requires effort; however, it has potential to reap valuable rewards, making healthcare improvements more effective, inclusive, and healing.

\section{Acknowledgments}

The authors wish to thank Jeffrey S. Stewart for his contributions and feedback on this topic and manuscript.

Disclosures: The authors have nothing to disclose.

\section{References}

1. Friedman S. Total Leadership: Be a Better Leader, Have a Richer Life (With New Preface). Boston, Massachusetts: Harvard Business Review Press; 2014.

2. Minkler M. Community-based research partnerships: challenges and opportunities. J Urban Health. 2005;82(2 Suppl 2):ii3-12. https://doi.org/10.1093/ jurban/jti034

3. Ponte PR, Conlin G, Conway JB, et al. Making patient-centered care come alive: achieving full integration of the patient's perspective. J Nurs Adm. 2003;33(2):82-90. https://doi.org/10.1097/00005110-200302000-00004

4. O'Leary KJ, Chapman MM, Foster S, O'Hara L, Henschen BL, Cameron KA. Frequently hospitalized patients' perceptions of factors contributing to high hospital use. J Hosp Med. 2019;14(9):521-526. https://doi.org/10.12788/ jhm.3175

5. Velez CM, Nicolaidis C, Korthuis PT, Englander H. "It's been an experience, a life learning experience": a qualitative study of hospitalized patients with substance use disorders. J Gen Intern Med. 2017;32(3):296-303. https://doi. org/10.1007/s11606-016-3919-4 